\title{
Associação entre (in) satisfação com a imagem corporal, estado nutricional e nível de coordenação motora em crianças e adolescentes de projetos esportivos
}

\author{
Association between body image satisfaction, nutrirional \\ status and motor cordination level in children and teens \\ participating in sports projects
}

Marcos Roberto Brasil', Vinicius Machado de Oliveira², Zacarias Chumlhak², Bruna Janaina Estevão², Thais Renata da Silva², Schelyne Ribas da Silva²

'Faculdade Guairacá, Bolsista Universidade Sem Fronteiras (USF), Guarapuava, PR, Brasil.

2Universidade Estadual do Centro-Oeste (Unicentro), Bolsista Universidade Sem Fronteiras (USF), Guarapuava, PR, Brasil.

Recebido em: fevereiro 2015 / Aceito em: maio 2015

edfmarcos-brasil@bol.com.br

\section{RESUMO}

Objetivo: identificar o nível de (in) satisfação corporal, estado nutricional e coordenação motora de crianças e adolescentes participantes de projetos esportivos e suas possíveis associações. Método: a amostra foi composta por 84 participantes de projetos esportivos com faixa etária entre nove a 16 anos, de ambos os sexos. A coordenação motora foi avaliada pelo teste KTK (Körperkoordinationstest Für Kinder), o estado nutricional através do índice de massa corporal (IMC) e a imagem corporal pelas silhuetas. Para a análise estatística, foram utilizados média e desvio padrão, teste $T$, frequência absoluta e relativa e o teste qui-quadrado. Resultados: identificou-se que 21,4\% apresentam sobrepeso; na classificação do desempenho da coordenação motora, $27,4 \%$ apresentam perturbações na coordenação e 13,1\% insuficiência na coordenação; já em relação à imagem corporal, 63,1\% apresentam insatisfação com sua silhueta. Considerações finais: a coordenação motora não esteve associada com o IMC; por outro lado, a insatisfação corporal apresentou relação com o estado nutricional, sendo que os escolares apresentam uma imagem corporal distorcida, uma vez que a maior parte daqueles que apresentam excesso de peso estão insatisfeitos com seu corpo pela magreza.

Palavras-Chave: Imagem Corporal; Atividade Motora; Crianças; Adolescentes.

\section{ABSTRACT}

Objective: identify levels of body dissatisfaction and satisfaction, nutritional status and motor coordination of children and adolescents participating in sports projects and their possible associations. Method: sample was comprised of 84 participants of sports projects aged between nine to 16 years, both genders. Motor coordination was assessed by KTK test (Körperkoordinationstest Für Kinder), nutritional status through the body mass index (BMI) and body image by the silhouettes. For statistical analysis average and standard deviation were used, besides $T$ test, absolute and relative frequency and the Chi-square test. Results: $21.4 \%$ of subjects presented overweight. In the performance of motor coordination, $27.4 \%$ showed coordination disturbances, and $13.1 \%$ coordination failure. Regarding body image, $63.1 \%$ presented dissatisfaction with your silhouette. Closing remarks: motor coordination was not associated with the BMI. On the other hand, body dissatisfaction was associated to nutritional status. Subjects have a distorted body image since most of those who are overweight are dissatisfied with your body due to thinness.

Keywords: Body Image; Motor Activity; Children; Adolescent. 


\section{INTRODUÇÃO}

A imagem corporal pode interferir em diferentes etapas do ser humano; durante a infância, com a construção da autoimagem, a percepção de ideais de beleza passa a ser de suma importância, podendo acarretar consequência durante a adolescência e se agravar durante a vida adulta. ${ }^{1} \mathrm{~A}$ imagem corporal pode ser considerada uma construção multidimensional que vai sendo formada juntamente com o indivíduo e fatores sociais, fisiológicos, psicológicos e ambientais podem alterar a visão tida do corpo. $^{2}$ A busca pelo corpo considerado ideal e a aparência física parecem ser uma das preocupações mais evidentes entre crianças e adolescentes, pois estudos com esta população revelam grande insatisfação corporal. ${ }^{3-8}$ A falta de atividade física está associada ao elevado índice de massa corporal, ${ }^{9}$ o qual interfere também no desempenho motor das crianças. ${ }^{10}$

O desempenho motor, por sua vez, pode ser definido como um comportamento observável do indivíduo em relação à execução de alguma atividade motora que, na atualidade, vem sendo reduzidas drasticamente, normalmente trocadas por televisão, computador, vídeo game, entre outros, beneficiando um estilo de vida mais sedentário. Dessa forma, para assegurar que crianças e adolescentes tenham um desenvolvimento satisfatório, é de suma importância apresentar ambientes ricos em estímulos, pois quanto mais instigadas forem essas habilidades, mais rico será o seu acervo motor. ${ }^{10}$ Portanto, o objetivo do presente estudo foi identificar o nível de (in) satisfação corporal, estado nutricional e coordenação motora de crianças e adolescentes participantes de projetos esportivos e suas possíveis associações.

\section{MÉTODO}

\section{Amostra}

A amostra foi composta por 84 participantes de projetos esportivos com faixa etária entre nove a 16 anos, de ambos os sexos, do município de Guarapuava-PR, os quais foram divididos em G1 (Grupo 1): nove a 12 anos e G2 (Grupo 2): 13 a 16 anos. A seleção dos participantes foi por voluntariedade. Inicialmente, a pesquisa foi aprovada pelo Comitê de Ética em Pesquisa (CEP), sob parecer 487.685/2013. Após a aprovação pelo CEP e o recolhimento dos termos livre esclarecidos, devidamente assinados pelos pais ou responsáveis pelos participantes, as avaliações foram realizadas em um núcleo do projeto esportivo.

\section{Instrumentos de Medida}

Avaliação do estado nutricional: foi baseada no índice de massa corporal (IMC $=$ peso $\mathrm{kg} /$ estatura $\mathrm{m}^{2}$ ). A medida da massa corporal foi realizada em uma balança da marca $\left(\mathrm{WELMY}^{\circ}\right)$, com precisão de $0,1 \mathrm{~kg}$ e a medida da estatura foi obtida em um estadiômetro de madeira com precisão de $0,1 \mathrm{~cm}$. A avaliação do estado nutricional dos participantes foi baseada no índice de massa corporal (IMC), o qual foi classificado seguindo os pontos de corte para idade e sexo da Organização Mundial da Saúde. ${ }^{11}$

Avaliação da imagem corporal: foram utilizadas as silhuetas proposta por Kakeshita..$^{12} \mathrm{O}$ grau de satisfação ou insatisfação com a imagem corporal foi determinado pela diferença entre as silhuetas desejada e a atual. A partir da diferença, foram considerados satisfeitos os participantes que apresentaram resultado zero (silhueta atual=desejada), insatisfeitos por magreza quando o resultado apresentou diferença positiva (silhueta atual >desejada), e, insatisfeitos por excesso de peso os avaliados que apresentaram a "meta" de obter corpo ou silhueta maior do que à atual (silhueta atual $<$ desejada).

Avaliação da coordenação motora: foi aplicado o teste de coordenação corporal para crianças (Körperkoordinationstest Für Kinder - KTK). ${ }^{13} \mathrm{O}$ teste é composto por quatro tarefas motoras: Trave de Equilíbrio; Saltos Monopedais; Saltitos Laterais e Transferência sobre plataformas. Para a determinação dos coeficientes motores obtidos pelos participantes, foram utilizados os valores normativos propostos por Gorla, Duarte e Montagner. ${ }^{14}$ Os somatórios destes valores resultam em um quociente motor geral e na classificação do nível de desempenho da coordenação motora, representado na Tabela 1.

Tabela 1 - Classificação do KTK.

\begin{tabular}{lccc}
\hline Quociente motor & Classificação & DP & $\%$ \\
\hline $131-145$ & Coordenação Alta & +3 & $99-100$ \\
$116-130$ & Coordenação Boa & +2 & $85-98$ \\
$86-115$ & Coordenação Normal & +1 & $17-84$ \\
$71-85$ & Perturbações na Coordenação & -2 & $3-16$ \\
$56-70$ & Insuficiência na Coordenação & -3 & $0-2$ \\
\hline KTK: Körperkoordinationstest Für Kinder; DP: Desvio Padrão. &
\end{tabular}

\section{Análise de Dados}

Para a análise estatística, foram utilizados valores médios e desvio padrão, teste $T$ para amostras independentes, frequência absoluta e relativa e o teste de qui-quadrado $\left(X^{2}\right)$, no pacote estatístico SPSS 21.0, considerando nível de significância para $p<0,05$.

\section{RESULTADOS}

Observa-se, na tabela 2, que o IMC difere significativamente $(p=0,013)$ entre as faixas etárias, sendo superior no G2. Não foram observadas diferenças entre os grupos na avaliação do quociente motor.

Tabela 2 - Média e Desvio Padrão do Índice de Massa Corporal e Quociente Motor.

\begin{tabular}{lccccc}
\hline & \multicolumn{2}{c}{ G1 } & \multicolumn{2}{c}{ G2 } & \\
\cline { 2 - 5 } Variáveis & Média & (DP) & Média & (DP) & Valor de $\boldsymbol{p}$ \\
\hline IMC & 18,8 & $(3,6)$ & 20,7 & $(3,2)$ & 0,013 \\
Quociente & 85,1 & $(13,6)$ & 89,8 & $(15,6)$ & 0,151 \\
Motor & & & & & \\
\hline
\end{tabular}

IMC: Índice de Massa Corporal, DP: Desvio Padrão; G1: 9 a 12 anos; G2: 13 a 16 anos

Identificou-se, na amostra estudada, que 21,4\% estão acima do peso; além disso, na avaliação do teste KTK, 27,4\% apresentam perturbações na coordenação e $13,1 \%$ insuficiência na coordenação. Com relação à percepção da imagem corporal, 46,4\% estão insatisfeitos pela magreza e $16,7 \%$ pelo excesso de peso. 
Tabela 3 - Frequência absoluta e relativa das variáveis.

\begin{tabular}{llll}
\hline & Variáveis & $\mathbf{n}$ & $\%$ \\
\hline Grupo & 09 a 12 anos & 36 & $(42,9)$ \\
& 13 a 16 anos & 48 & $(57,1)$ \\
\hline IMC & Eutrófico & 66 & $(78,6)$ \\
& Acima do Peso & 18 & $(21,4)$ \\
\hline Classificação & Normal & 50 & $(59,5)$ \\
KTK & Perturbações na Coordenação & 23 & $(27,4)$ \\
& Insuficiência na Coordenação & 11 & $(13,1)$ \\
\hline Classificação & Satisfeito & 31 & $(36,9)$ \\
Imagem Corporal & Insatisfeito por Magreza & 39 & $(46,4)$ \\
& Insatisfeito por Excesso de Peso & 14 & $(16,7)$ \\
\hline
\end{tabular}

IMC: Indice de Massa Corporal; KTK: Körperkoordinationstest Für Kinder.

Tabela 4 - Frequência absoluta e relativa das variáveis.

\begin{tabular}{|c|c|c|c|c|}
\hline Variáveis & $\begin{array}{l}\text { Normal } \\
\text { n (\%) }\end{array}$ & $\begin{array}{c}\text { Perturbação na Coordenação } \\
\text { n (\%) }\end{array}$ & $\begin{array}{c}\text { Insuficiência na Coordenação } \\
\text { n (\%) }\end{array}$ & $p$ \\
\hline \multicolumn{5}{|l|}{ Eutrófico } \\
\hline G1 & $14(50,0)$ & $11(39,3)$ & $3(10,7)$ & 1,000 \\
\hline G2 & $27(71,1)$ & $7(18,4)$ & $4(10,5)$ & 0,286 \\
\hline \multicolumn{5}{|c|}{ Acima do Peso } \\
\hline G1 & $4(50,0)$ & $3(37,5)$ & $1(12,5)$ & 1,000 \\
\hline G2 & $5(50,0)$ & $2(20,0)$ & $3(30,0)$ & 0,286 \\
\hline \multicolumn{5}{|l|}{ Satisfeito } \\
\hline G1 & $7(53,8)$ & $5(38,5)$ & $1(7,7)$ & 0,687 \\
\hline $\mathrm{G} 2$ & $14(77,8)$ & $3(16,7)$ & $1(5,6)$ & 0,615 \\
\hline \multicolumn{5}{|c|}{ Insatisfeito por Magreza } \\
\hline G1 & $8(50,0)$ & $7(43,7)$ & $1(6,3)$ & 0,687 \\
\hline $\mathrm{G} 2$ & $13(56,5)$ & $5(21,7)$ & $5(21,7)$ & 0,615 \\
\hline \multicolumn{5}{|c|}{ Insatisfeito por Excesso de Peso } \\
\hline G1 & $3(42,9)$ & $2(28,6)$ & $2(28,6)$ & 0,687 \\
\hline $\mathrm{G} 2$ & $5(71,4)$ & $1(14,3)$ & $1(14,3)$ & 0,615 \\
\hline
\end{tabular}

G1: 9 a 12 anos; G2: 13 a 16 anos; diferencas significativas para $p \leq 0,05$.

Tabela 5 - Associação entre Faixa Etária, Índice de Massa Corporal e Imagem Corporal.

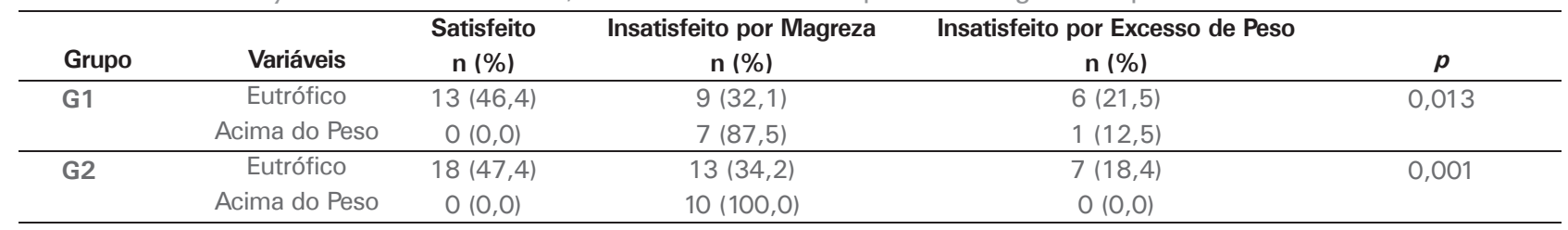

G1: 9 a 12 anos; G2: 13 a 16 anos; diferenças significativas para $p \leq 0,05$

$\mathrm{Na}$ a associação do KTK com IMC e imagem corporal, divididos por faixa etária (Tabela 4), não foram observadas diferenças significativas. Porém, ressalta-se que, entre os escolares acima do peso, o percentual de alterações na coordenação (perturbação e insuficiência) é superior, na comparação com o grupo eutrófico, para o G2.

A associação entre o IMC e a imagem corporal (Tabela 5) demonstra que existe uma associação significativa entre essas duas variáveis, em ambos os grupos. Dessa forma, observa-se que existe uma distorção da imagem corporal, uma vez que os escolares acima do peso estão insatisfeitos com seu corpo pela magreza.

\section{DISCUSSÃO}

Nossos resultados em relação à insatisfação da imagem corporal $(63,1 \%)$ são inferiores ao estudo que investigou escolares de sete a 17 anos do município de
Cascavel-PR e encontrou $74,7 \%$ de insatisfação corporal, ${ }^{3}$ bem como em escolares do sexto ano do município de Caxias do Sul-RS com idade entre 11 a 14 anos, com $71,5 \%$ de descontentamento com a imagem corporal. ${ }^{6}$ Porém, nossos dados são semelhantes aos resultados relatados em crianças e adolescentes com idade de oito a 16 anos participantes de clubes esportivos do município de Londrina-PR, no qual obteve $61,8 \%$ de insatisfação com a imagem, ${ }^{5}$ assim como em estudantes do $5^{\circ}$ ano do ensino fundamental até o $3^{\circ}$ ano do ensino médio do município de Saudade-SC, ${ }^{4}$ com idade entre 11 a 17 anos, que demonstrou uma insatisfação de $60 \%$. Por outro lado, entre os adolescentes de 14 a 18 anos do sexo feminino, regularmente matriculadas em escolas públicas e particulares do município de Belo Horizonte-MG, a insatisfação corporal encontrada foi muito inferior $(27,2 \%),^{7}$ sendo semelhante aos dados encontrados entre adolescentes do sexo feminino com 
idade entre 11 a 13 anos, estudantes de escolas públicas do município de Santa Maria-RS (25,3\%). ${ }^{8}$ Tal diferença pode ser explicada pelo fato de que cada região possui uma diversidade cultural, a faixa etária estuda e também a utilização de silhuetas diferentes do estudo.

Para a associação da imagem corporal e faixa etária, o G1 eutróficos apresentou uma insatisfação por excesso de peso de 21,4\%, valor maior aos eutróficos do $\mathrm{G} 2$, com 18,4\%; tais resultados divergem do estudo com escolares de 14 a 17 anos, em que 48,0\% desejam aumentar a silhueta (insatisfeito por magreza), assim como o grupo de 11 a 13 anos $(41,0 \%) .^{3}$ Quando confrontadas as variáveis insatisfação com imagem corporal e estado nutricional, entre os participantes classificados como eutróficos, 46,9\% estão satisfeitos com sua imagem corporal, sendo $19,9 \%$ de insatisfação por excesso de peso e 33,1\% por magreza. Em Londrina-PR, 64\% dos classificados como eutróficos estão satisfeitos e $36 \%$ insatisfeitos com sua imagem corporal; já os com excesso de peso representam $38,2 \%$ de satisfação e $61,8 \%$ de insatisfação com a imagem corporal. ${ }^{5}$ Estudo realizado em Santa Maria-RS revela que a insatisfação manteve-se associada ao IMC, sendo que os adolescentes com excesso de peso apresentaram 2,64 vezes mais chances de estarem insatisfeitas com a imagem corporal em relação aqueles com IMC normal, ${ }^{8}$ assim como demonstrado em Caxias do Sul-RS, sendo encontrada relação significativa entre excesso de peso e insatisfação com a imagem corporal, em que os escolares com excesso de peso apresentaram 3,84 vezes mais chances de estarem insatisfeitos em relação aos escolares com peso adequado ou baixo peso. ${ }^{6}$ Resultados superiores foram encontrados em Saudade-SC, em que rapazes com alto IMC apresentam 4,24 e moças 6,81 vezes mais chances de insatisfação corporal. ${ }^{4}$ Dessa forma, os estudos mostram que o IMC está associado com a insatisfação corporal; porém, Glaner et al. destacam que a adiposidade corporal, por meio da somatória de espessura de duas dobras cutâneas (regiões do tríceps e perna medial), pode ser um melhor indicador antropométrico de insatisfação corporal do que o IMC, pelo fato das dobras cutâneas ser um estimador da adiposidade corporal, enquanto que o IMC indica somente a quantidade de massa por superfície. ${ }^{4}$

A associação da imagem corporal em relação à classificação motora geral demonstra que não foram observados níveis de coordenação além da normalidade, semelhantes aos estudos realizados em Cianorte-PR ${ }^{10} \mathrm{e}$ em Umuarama-PR, ${ }^{14}$ os quais também não encontram níveis acima da faixa de normalidade; em Portugal, estudos $^{15,16}$ mostram que o nível de desempenho da coordenação motora aumenta a medida que a faixa etária aumenta. Valdivia et al. ${ }^{17}$ sugerem que este resultado pode estar relacionado aos efeitos do processo de crescimento e maturação.

\section{CONSIDERAÇÕES FINAIS}

Observou-se, no presente estudo, que é elevado o percentual de escolares com perturbações ou insuficiência na coordenação e com excesso de peso. Não foi encontrada relação entre a coordenação motora e o IMC; porém, observa-se associação entre insatisfação corporal com o estado nutricional real, sendo que os escolares avaliados apresentam uma imagem corporal distorcida, já que a maior parte daqueles que estão com excesso de peso estão insatisfeitos com seu peso corporal pela magreza.

\section{AGRADECIMENTOS}

A equipe agradece as crianças, adolescente e aos pais/responsável pela participação desta pesquisa.

Fonte financiadora do projeto: o projeto possui financiamento externo e duração de 12 meses Edital 01/2013 do Programa de Extensão Universidade sem Fronteiras, Sub-Programa Apoio às Licenciaturas, da Secretaria de Estado da Ciência, Tecnologia e Ensino Superior do Paraná - SETI.

\section{REFERÊNCIAS}

1. Smolak L, Levine MP, Thompson JK. The Use of the Sociocultural Attitudes Towards Appearance Questionnaire with Middle School Boys and Girls. Sociocult Attitudes 2001; 29(1): 216-23. DOI: http://dx.doi.org/10.1002/1098-108X(2 00103)29:2\%3C216::AIDEAT1011\%3E3.0.CO;2-V.

2. Fernandes AER. Avaliação da Imagem Corporal, hábitos alimentares em crianças e adolescentes de escolas públicas e particulares de Belo Horizonte. Universidade Federal de Minas Gerais; 2007. p. 143.

3. Fidelix YL, Minatto G, Santos KD, Petroski EL. Dados sociodemográficos, estado nutricional e maturação sexual de escolares do sexo masculino: exposição à insatisfação com a imagem corporal. Rev Educ Física/UEM 2013; 24(1): 83-92. DOI: http://dx.doi.org/10.4025/reveducfis.v24.1.17353.

4. Glaner MF, Pelegrini A, Cordoba CO, Pozzobon ME. Associação entre insatisfação com a imagem corporal e indicadores antropométricos em adolescentes. Rev Bras Educ Física e Esporte 2013; 27(1): 129-36.

5. Schubert A, Januário RSB, Casonatto J, Sonoo CN. Imagem corporal, estado nutricional, força de resistência abdominal e aptidão cardiorrespiratória de crianças e adolescentes praticantes de esportes. Rev Paul Pediatr 2013; 31(1): 71-6.

6. Finato S, Rech RR, Migon P, Gavineski IC, Toni V de, Halpern R. Insatisfação com a imagem corporal em escolares do sexto ano da rede municipal de Caxias do Sul, no Rio Grande do Sul. Rev Paul Pediatr 2013; 31(1): 65-70.

7. Beling MTC. A auto-imagem corporal e o comportamento alimentar de adolescentes do sexo feminino em Belo Horizonte, MG. Universidade Federal de Minas Gerais; 2008. p. 1-165.

8. Martins CR, Pelegrini A, Petroski EL. Insatisfação com a imagem corporal e relação com estado nutricional , adiposidade corporal e sintomas de anorexia e bulimia em adolescentes. Rev Psiquiatr 2010; 32(48): 19-23.

9. Silva KS da, Nahas MV, Hoefelmann LP, Lopes A da S, Oliveira ES De. Associações entre atividade física, índece de massa corporal e comportamentos sedentários em adolescente. Rev Bras Epidemiol 2008; 11(1): 159-68.

10. Carminato RA. Desempenho motor de escolares através da bateria de teste KTK. Universidade Federal do Paraná; 2010. p. 99.

11. Onis M De, Onyango AW, Borghi E, Siyam A, Nishida C, Siekmann J. Development of a WHO growth reference for school-aged children and adolescents. Bull World Health 
Organ 2007; 85(9): 660-7. DOI: http://dx.doi.org/10.2471/ BLT.07.043497.

12. Kakeshita IS. Adaptação e validação de Escalas de Silhuetas para crianças e adultos brasileiros. 2008. p. 96.

13. Kiphard J., Schiling F. Körperkoordinationstest für kinder, KTK. 1974.

14. Gorla JI, Duarte E, Montagner PC. Avaliação da coordenação motora de escolares da área urbana do Município de UmuaramaPR Brasil. Rev Bras Ciência e Mov 2009; 16(2): 57-65.

15. Lopes LCO. Actividade Física, recreio escolar e desenvolvimento motor. Estudos Exploratórios em Crianças do 1o Ciclo do Ensino Básico. Universidade do Minho; 2006. p. 94.

16. Deus RKBC de, Bustamante A, Lopes VP, Seabra AT e, Silva RMG da, Maia JAR. Modelação longitudinal dos níveis de coordenação motora de crianças dos seis aos 10 anos de idade da Região Autônoma dos Açores , Portugal. Rev Bras Educ Física e Esporte 2010; 24(2): 259-73.

17. Valdivia $A B$, Cartagena LC, Távara NE, Seabra AT e, Silva RMG da, Maia JAR. Coordinacón Motora: influencia de la edad, sexo, estatus socio-económico y nivels de adiposidad en níños peruanos. Rev Bras Cineantropometria Desempenho Hum 2008; 10(1): 25-34. 